\title{
Early Effects of the COVID-19 Pandemic on Physical Activity in U.S. Adults
}

\author{
Genevieve F. Dunton ${ }^{1,2}$, PhD, MPH, Shirlene D. Wang ${ }^{1}$, Bridgette Do ${ }^{1}$, MPH, \\ Jimikaye Courtney ${ }^{3}, \mathrm{PhD}$
}

\begin{abstract}
Affiliations: ${ }^{1}$ Department of Preventive Medicine, Keck School of Medicine, University of Southern California; ${ }^{2}$ Department of Psychology, Dornsife College of Letters, Arts, and Sciences, University of Southern California; and ${ }^{3}$ Department of Health and Exercise Science, Colorado State University
\end{abstract}

Address correspondence to: Genevieve F. Dunton, $\mathrm{PhD}$, MPH, Professor, Department of Preventive Medicine, Keck School of Medicine, University of Southern California, 2001 N. Soto St., Los Angeles, CA, 90032, USA; Email: dunton@usc.edu; Phone: 323-442-8224, Fax: 323$442-8201$ 


\begin{abstract}
Purpose: COVID-19 restrictions, social-distancing, and quarantining may inhibit U.S. adults from attaining recommended levels of physical activity. The aims of this study were to examine the early impact of the COVID-19 pandemic on physical activity in U.S. adults during the first month of COVID-19 restrictions, and whether changes in physical activity levels differed by demographic characteristics.
\end{abstract}

Method: U.S. adults completed an online survey between April 10-May 5, 2020. Participants were recruited through convenience sampling from social media and mass emails. The analytic sample included 262 adults from 30 states and the District of Columbia. Using the short-form International Physical Activity Questionnaire (IPAQ), participants reported minutes of vigorous intensity, moderate intensity, and walking physical activity for a typical week in February 2020 (pre-COVID-19) and for the past 7 days (early-COVID-19). Participants also reported locations (e.g., home/garage, parks/trails, gyms/fitness centers) were they had performed physical activity over the past 7 days and demographic characteristics.

Results: Participants (82\% female, 20\% Hispanic, 41\% overweight/obese) ranged in age from 18-79 $(\mathrm{M}=32.9, \mathrm{SD}=12.3)$ years. On average, significant reductions in vigorous intensity ($61.7 \mathrm{~min} /$ week, 37\% decrease), moderate intensity (-84.1 $\mathrm{min} /$ week, $47 \%$ decrease), and walking (-104.4 min/week, 33\% decrease) physical activity were observed during the early-COVID 19 period vs. pre-COVID-19 period. Reductions in vigorous intensity physical activity were greater for adults who were not working. Reductions in walking physical activity were greater for adults who were Hispanic or resided in lower income households.

Conclusion: Vulnerable population sub-groups may be harder hit by the COVID-19 pandemic in terms of its impact on health-related behaviors. Communication, programmatic, and 
policy efforts should be directed towards promoting physical activity in these at-risk groups during the COVID-19 pandemic.

Key words: vigorous activity; moderate activity; walking; Hispanic; income; employment status 


\section{Introduction}

COVID-19, the respiratory disease caused by the SARS-CoV-2 virus, has been declared a pandemic by the World Health Organization and a national emergency in the United States of America (U.S.). Originating in Wuhan, China in late 2019, COVID-19 first spread to countries in Asia and Europe in February, and then quickly to North America and around the globe in March and April, 2020. As of the date this article was written (May 13, 2020), there were 1.3 million COVID-19 cases and 80,820 related deaths recoded by the U.S. Centers for Disease Control and Prevention (1).

In order to limit the spread of COVID-19, state governments in the U.S. issued "Shelterin-place" or "Stay-at-home" orders starting between March 19 - April 3, 2020 (2). Although a few states rescinded or ended these orders in late April and early May, most states extended the expiration dates through May 15 - May 29, 2020. "Shelter-in-place" and "Stay-at-home" orders vary in scope from state to state but generally require "non-essential" businesses to close their physical offices or storefronts and continue their operations remotely. These guidelines require residents to remain at home except when performing essential activities including purchasing groceries and seeking medical treatment. In most states, local governments ordered all restaurants and bars to halt dine-in service, and for gyms/fitness facilities, theatres, bowling alleys, hair/nail salons, and other non-essential business to temporarily close. Orders do not completely prohibit people from leaving their homes, as residents are allowed to take walks and in outdoor spaces as long as they maintain social distancing of at least 6 feet away from people not in their households. Starting mid-March, schools closed in all 50 states with many states extending schools closures through the end of the 2019-2020 school year $(3,4)$. Federal, state, and local public parks, trails, and beaches were also closed in many jurisdictions starting mid-to- 
late March with some re-openings occurring in late April and early May, and most are scheduled for later dates.

Despite the public health necessity of these social-distancing measures in order to slow the spread of COVID-19 and ensure that medical facilities have adequate resources to address needs, they may impose restrictions on individuals' ability to engage in sufficient levels of physical activity in order to maintain health and prevent further disease. The Physical Activity Guidelines for Americans recommend that adults should engage in at least 150 minutes of moderate-intensity or 75 minutes of vigorous-intensity physical activity per week to reduce risks of obesity, cancer, heart disease, diabetes, and other chronic conditions (5). However, prepandemic levels of adherence to these guidelines were already less than desired for population health. According to estimates, approximately $65 \%$ of U.S. adults achieved these physical activity guidelines in 2015-2016 when assessed through self-report (6), and estimates of adherence to national physical activity guidelines may much lower when assessed through accelerometer (7). Participation rates in physical activity are not equal across all segments of the populations, with lower levels of physical activity in women, older adults, Hispanics, and those with lower household incomes (6).

Restrictions and social-distancing measures introduced by the COVID-19 pandemic may inhibit U.S. adults from attaining recommended levels of physical activity $(8,9)$. More than 70 million Americans regularly access gyms and fitness facilities (10), indicating that closure of these businesses may have a profound effect on physical activity behaviors. Furthermore, restricted access to public parks, trails, and beaches may eliminate low- and no-cost opportunities for outdoor physical activity. Potentially, one of the more significant impacts on physical activity levels during the COVID-19 pandemic may be the changes in daily routines, 
habits, mobility, and commuting patterns of U.S. adults. By limiting reasons to leave the house, incidental physical activity (e.g., during errands or chores) and active transportation (e.g., biking to work, walking to public transit) may decline dramatically. Preliminary data on the impact of COVID-19 restrictions suggests that physical activity among U.S. adults was reduced between $32 \%$ and $48 \%$ through the first week of April $2020(11,12)$. Although alternative forms of leisure-time physical activity, such as virtual fitness/exercise classes and neighborhood-based walking, have the potential to replace activity taking place at facilities that have been closed during the pandemic (e.g., gyms/fitness facilities, parks/trails), there may be enormous disparities in access to these opportunities based on sociodemographic and economic characteristics.

The overall goal of the current study was to investigate the early impact of the COVID19 pandemic on activity levels among U.S. adults during the first full month that "Shelter-inplace" and "Stay-at-home" orders were in place. The first objective was to determine whether levels of vigorous, moderate, and walking physical activity significantly declined from the preCOVID-19 period (February 1-29, 2020) to the early-COVID-19 period (April 3 - May 5, 2020). The second objective was to examine whether changes in physical activity levels differed by sociodemographic characteristics (e.g., age, sex, ethnicity, annual household income, current work/school status). Ancillary goals were to examine whether locations of physical activity and the use of remote/streaming services for physical activity also differed by sociodemographic characteristics. Given emerging evidence of the potential immune-protective effect of cardiorespiratory exercise against complications of COVID-19 $(13,14)$ combined with concerns that temporary lapses in physical activity can extend to permanent disengagement with the behavior (15), understanding the early effects of the COVID-19 pandemic on physical activity levels in U.S. adults is an important public health issue. 


\section{Methods}

\section{Study Design}

We adopted a prospective survey design to assess the early effects of the COVID-19 pandemic on physical activity among U.S. adults by using online surveys. A baseline online survey was completed between April 10 - May 5, 2020, and a follow-up online survey is scheduled to occur within 6-12 months. Following the baseline survey, some participants completed 28 subsequent days of mobile phone-based ecological momentary assessment with morning and evening surveys each day. The current analyses focus on data collected from the baseline online survey.

\section{Recruitment and Participants}

A convenience sampling strategy, focused on recruiting general populations of adults living in the U.S. during the COVID-19 pandemic, was utilized. In order to minimize in-person interactions, potential respondents were electronically invited through various social media platforms (e.g., Facebook, Twitter, Reddit, Linked In) and university-based email list servs of students, faculty, and staff. Inclusion criteria were as follows: 18 years or older, able to speak and read English, live in the U.S., and own and regularly use an Android or iPhone smartphone that they are willing to use to complete app-based surveys for the duration of the study. Exclusion criteria were as follows: enrolled in another study related to physical activity behaviors, including studies monitoring physical activity, intervening on physical activity, or examining the effects of wearable fitness trackers. Individuals were directed to an online screening form directly from an email or social media post. Once eligibility was determined, individuals agreed to participate through on online anonymous information sheet that described the study procedures, risks, and benefits. The Institutional Review Board of the University of 
Southern California determined that the study procedures presented no more than minimal risk and approved it as exempt from full review (HS-20-00304).

\section{Procedures}

Participants completed the online screening form, information sheet, and baseline survey in English through an online survey platform Research Electronic Data Capture, which conforms to HIPAA security requirements for the protection of data $(16,17)$. Baseline data collection took place over 26 days (April 10 - May 5, 2020), which cover the first full month after most U.S. states issued "Shelter-in-place" or "Stay-in-home" orders. The baseline survey took approximately 30 minutes to complete. Participants had the option to complete it either on their mobile phone, tablet, or desktop device. Upon completion of the baseline survey, participants were eligible to be entered into a lottery to win one of ten $\$ 50$ gift cards.

\section{Measures}

In order to guide the development of the survey, recently published questionnaires on COVID-19 symptoms, diagnoses, and impacts were reviewed (18). Authors included additional questions related to studying the impact of the COVID-19 pandemic on physical activity. The structured survey consisted of questions that covered several areas including: health history; potential contact history with COVID-19 in the past 14 days; current physical symptoms of illness; COVID-19 diagnosis and hospital admission history; changes in employment and caregiving duties due to COVID-19; self-quarantining and social-distancing practices; impact of COVID-19 on daily life; positive and negative affect, general perceived stress, and COVID-19 related stress; physical activity levels and sitting time in February 2020 and in the past 7 days; physical activity stage of change, motivations, habits, and identity; locations of physical activity and use of remote/streaming services for physical activity in the past 7 days; demographic; and 
U.S. zip code. The current analyses utilized data on physical activity levels, physical activity locations, and demographics.

Physical activity. Participants completed the International Physical Activity

Questionnaire Short Form (IPAQ-SF) (19) twice in the baseline survey. The IPAQ is one of the most widely used report-based physical activity instruments and has demonstrated acceptable reliability and validity across a range of populations (20-22). The first time, the instructions asked individuals to "Think about the time you spent being physically active during a typical week in February 2020 (BEFORE THE COVID-19 PANDEMIC).” The second time, the instructions asked individuals to "Think about the time you typically spent being physically active in the last 7 days PRIOR TO TODAY." During both times, participants reported on levels of vigorous intensity activity, moderate intensity activity, and walking activity. Vigorous intensity activities were described as "activities that take hard physical effort and make you breathe much harder than normal" and included examples such as heavy lifting, digging, aerobics, or fast bicycling. Moderate intensity activities were described as "activities that take moderate physical effort and make you breathe somewhat harder than normal" such as carrying light loads, bicycling at a regular pace, or doubles tennis. Walking activities were described as including "walking at work and at home, walking to travel from place to place, and any other walking that you have done solely for recreation, sport, exercise, or leisure." For all three types of physical activity, individuals reported the number of days per week that they performed the targeted activity for at least 10 minutes at a time. They then reported how much time they usually spent on one of those days doing the targeted physical activity. Data were cleaned following the IPAQ scoring protocol (23). Outliers (with a z-score $\geq 3$ standard deviations above the mean) were excluded from the analyses. 
Physical activity locations. Participants were asked whether they were currently physically active for at least 30 minutes per week. If so, they were asked to indicate where (i.e., the types of locations in which) they did physical activity over the past 7 days, with instructions to choose all that apply from the following options: inside my home or garage, in my yard or driveway, on the sidewalks and roads in my neighborhood, on the sidewalks and roads outside my neighborhood, gym or fitness center, at a park or trail, at an outdoor sports facility (e.g., basketball/tennis court, baseball diamond, etc.).

Demographics. Participants reported their biological sex at birth (male vs. female), age (later categorized as 18-39 years, 40-59 years, and 60+ years), ethnicity (Hispanic vs. nonHispanic), race (Asian, Black, White, Mixed, Other), education level (completed high school/GED, some college, college graduate, graduate school degree), current work/school status (full-time employment [ $\geq 40$ hours/week], part-time employment $[<40$ hours/week], not working, in school only), and annual household income (later categorized as less than $\$ 27,000, \$ 27,000$ $\$ 59,999, \$ 60,000-\$ 99,999, \$ 100,000$ or more). Participants also reported their height (inches) and weight (pounds). Height was converted to meters, and weight was converted to kilograms. Body mass index (BMI; $\mathrm{kg} / \mathrm{m}^{2}$ ) was calculated, and weight status categories were created (underweight is $<18.5 \mathrm{~kg} / \mathrm{m}^{2}$, normal weight is $\geq 18.5 \mathrm{~kg} / \mathrm{m}^{2}$ and $<25 \mathrm{~kg} / \mathrm{m}^{2}$, overweight is $\geq 25$ $\mathrm{kg} / \mathrm{m}^{2}$ and $<30$, obese is $\geq 30 \mathrm{~kg} / \mathrm{m}^{2}$ ).

\section{Statistical Analyses}

Prior to data analyses, variables were screened for violations of statistical assumptions (e.g., normality, linearity). All three physical activity variables (i.e., vigorous intensity, moderate intensity, and walking) were positively skewed and thus subjected to square root transformations. Repeated-measures analysis of variance (ANOVAs) procedures examined within-subjects effects 
of time (pre-COVID-19 [February 1-29, 2020] vs. early-COVID-19 “Shelter-in-place”/“Stay-athome" orders [April 3- May 5, 2020]) on minutes of vigorous intensity, moderate intensity, and walking physical activity per week. A series of univariate ANOVAs tested the between-subjects effects of demographic factors (i.e., sex, age group, ethnicity, annual household income, work/school status, and weight status) on the physical activity variables pooled across the two time points. A mixed model repeated-measures analysis of variance procedure (ANOVA) examined the effects of demographic variables on the within-subject difference in physical activity between the two time points. Preliminary mixed models repeated measures ANOVAs determined which of the demographic variables should be included in the final models by testing them in separate models. Variables yielding a significant time (within-subjects) $\times$ demographic variable (between-subjects) interaction term were retained. In the final repeated-measures analyses, custom models were specified that tested the main effects of time and demographic variables, and all two-way interactions between time and demographic variables. In reporting the results of statistical analyses, effect sizes for the factors in the ANOVA model, partial eta squared $\left(\eta_{\mathrm{p}}^{2}\right)$ values, are provided. To address the ancillary goal of examining whether the likelihood of engaging in physical activity at the various locations also differed by demographic characteristics, logistic regressions were conducted with all demographic predictor variables entered simultaneously (with the exception of annual household income and current work/school status) and each location (yes vs. no) as the dependent variables in separate models.

Results

\section{Data Availability and Demographic Characteristics}

A total of 501 individuals expressed interest in the study and completed the screening questions. Of this number, $\mathrm{n}=10$ individuals were not eligible for living outside of the U.S., $\mathrm{n}=$ 
7 were not eligible because they did not own a compatible Android or iPhone smartphone, $\mathrm{n}=8$ were not eligible for being unwilling to comply with all of the study procedures, $n=12$ were not eligible for currently participating in another research study examining physical activity behaviors. A total of 471 individuals were eligible after completing the screener. Sixty-four individuals were no longer interested in the study after completing the screener, and 407 individuals consented for the study. One hundred and twenty-six cases were removed that had not yet completed the baseline survey at the time of data analysis $(n=281$ remaining $)$. An additional 19 cases with outliers ( $\geq 3$ standard deviations above the mean) on one or more of the physical activity variables (vigorous intensity, moderate intensity, walking) were excluded, leaving an analytic sample size of 262 . Table 1 shows the descriptive statistics for the demographic characteristics of the analytic sample. Individuals resided across 30 U.S. states and the District of Columbia. Participants ranged in age from 18-79 years old with an average age of $32.9(\mathrm{SD}=12.3)$ years. A majority of participants were female, non-Hispanic, and had graduated from college. Approximately half of participants reported working full-time. There was a range of annual household incomes represented, with about $17 \%$ reporting less than $\$ 27,000$ and about $40 \%$ reporting $\$ 100,000$ or more. Approximately $41 \%$ of the sample had BMIs classified as overweight or obese.

\section{Early Changes in Physical Activity During the early-COVID-19 Period}

Mean minutes per week of vigorous, moderate, and walking physical activity for the preCOVID-19 (February 2020) and early-COVID-19 (April 3- May 5, 2020) periods are shown in Table 2. The analysis yielded significant within-subject main effects of time on all three physical activity variables. On average, participants reported significant reduction in vigorous intensity (61.7 minutes/week), moderate intensity (-84.1minutes/week), and walking (-104.4 
minutes/week) physical activity during the early-COVID 19 period as compared to the preCOVID-19 period. These changes represent a $37.0 \%$ decrease in mean vigorous, $46.5 \%$ decrease in mean moderate, and $33.1 \%$ decrease in mean walking physical activity per week. For vigorous physical activity minutes per week, $49.8 \%$ of participants decreased, $26.7 \%$ remained at the same level, and $23 \% .5$ increased. For moderate intensity physical activity minutes per week, $50.8 \%$ of participants decreased, $26.4 \%$ remained at the same level, and 22.8\% increased. For minutes of walking physical activity per week, $54 \%$ of participants decreased, $12.5 \%$ remained at the same level, and $33.5 \%$ increased.

\section{Differences by Demographic Characteristics}

There were between-subject main effects of demographic characteristics on physical activity. Minutes of vigorous intensity physical activity per week differed by age group, $F(2,411)$ $=4.114, p=.017, \eta_{\mathrm{p}}^{2}=.032$ and work $/$ school status, $F(3,247)=2.724, p=.045, \eta_{\mathrm{p}}^{2}=.032$. Adults who were ages 18-39 reported more minutes of vigorous intensity physical activity per week than adults $>60$ years old, mean diff $=4.773, S E=1.686, p=0.15$, and adults who were working part-time reported more minutes of vigorous intensity physical activity per week than adults who were not working, mean $\operatorname{diff}=4.208, S E=1.55, p=0.43$. In addition, minutes of moderate intensity physical activity per week differed by work/school status, $F(3,250)=2.858, p$ $=.038, \eta_{\mathrm{p}}{ }^{2}=.003$. Adults who were working part-time reported marginally more moderate intensity physical activity per week than adults who were in school, mean diff $=3.265, S E=$ $1.279, p=0.68$. However, minutes of vigorous intensity physical activity per week did not differ by sex, ethnicity (Hispanic vs non-Hispanic), annual household income, or weight status. Also, minutes of moderate intensity physical activity per week did not differ by sex, age group, 
Hispanic ethnicity, annual household income, or weight status. Lastly, minutes of walking per week did not differ by sex, age group, Hispanic ethnicity, work/school status, or weight status.

Variable screening determined potential demographic characteristics to include in the final repeated-measures ANOVA. Results revealed that the size of the change in physical activity levels between pre-COVID-19 and early-COVID-19 differed by demographic characteristics. Work/school status had a significant effect on the size of the change in vigorous intensity physical activity minutes per week, $F(1,247)=3.111, \mathrm{p}=.027, \eta_{\mathrm{p}}{ }^{2}=.036$. In addition, annual household income had a significant effect of on the size of the change in moderate intensity physical activity minutes per week, $F(3,226)=3.037, p=.030, \eta_{\mathrm{p}}{ }^{2}=.039$. Hispanic ethnicity, $F(1,246)=5.639, p=.018, \eta_{\mathrm{p}}^{2}=.032$, and work/school status, $F(3,244)=2.704, p=.046, \eta_{\mathrm{p}}{ }^{2}=$ .032 , and annual household income, $F(3,220)=3.687, p=.013, \eta_{\mathrm{p}}^{2}=.048$ had significant effects on the size of the change in walking minutes per week. In contrast, neither sex, age group, nor weight status influenced the size of the change in any of the physical activity variables between pre-COVID-19 and early-COVID-19. Therefore, only Hispanic ethnicity, annual household income, and work/school status were included as between-subject factors in the final repeatedmeasures ANOVAs. Given the sizable association between annual household income and work/school status, chi-square $(\mathrm{df}=9)=34.880, p<.001$, these two variables were entered separately in the final models.

Results for the final models are shown in Tables 3-5. After controlling for Hispanic ethnicity, the size of the change in minutes of vigorous intensity physical activity per week between the pre-COVID-19 and early-COVID-19 periods differed by work/school status (See Table 3). In order to further explore the nature of this interaction, we compared the work/school status subgroups' standardized effect sizes for time $(24,25)$ with $90 \%$ confidence intervals due 
to the one-sided nature of the F-test (26). Adults who were not working $\left(\eta_{\mathrm{p}}^{2}=.444, \mathrm{CI}_{90 \%}=\right.$ $[.163,610])$ reported a greater decrease in vigorous intensity physical activity minutes per week as compared to adults who were working full-time $\left(\eta_{\mathrm{p}}^{2}=.061, \mathrm{CI} 90 \%=[.011, .137]\right)$. Figure 1 shows that the decrease in vigorous intensity physical activity minutes per week was greater for individuals who were not working. Table 4 shows that neither Hispanic ethnicity, annual household income, nor work/school status had effects on the size of the change in moderate intensity physical activity. Furthermore, after controlling for work/school status, the size of the change in minutes of walking physical activity per week differed by ethnicity (See Table 5). Hispanic adults $\left(\eta_{\mathrm{p}}{ }^{2}=.111, \mathrm{CI} 9_{0} \%=.009\right.$ to .257$)$ reported a greater decrease in walking physical activity minutes per week as compared to non-Hispanic adults $\left(\eta_{\mathrm{p}}{ }^{2}=.068, \mathrm{CI} 90 \%=\right.$ .021 to .131) (also see Figure 2). Lastly, after controlling for Hispanic ethnicity, the size of the change in minutes of walking physical activity per week differed by annual household income (See Table 5). Adults with annual household incomes between $\$ 27,00-\$ 59,999\left(\eta_{\mathrm{p}}{ }^{2}=.239\right.$, $\left.\mathrm{CI} 9_{0 \%}=[.089, .381]\right)$ reported a greater decrease in walking physical activity minutes per week as compared to adults with annual household incomes greater than or equal to $\$ 100,000\left(\eta_{\mathrm{p}}^{2}=\right.$ $\left..007, \mathrm{CI}_{90 \%}=[.000, .060]\right)($ also see Figure 3).

\section{Locations of Physical Activity}

During the early-COVID-19 period, the greatest proportion of physical activity was reported at home or in one's garage $(66.8 \%)$, followed by on the sidewalks or roads in one's neighborhood $(59.9 \%)$, at a park or trail $(23.3 \%)$, on the sidewalks or roads outside one's neighborhood (18.7\%), in one's yard or driveway (15.6\%), gym or fitness center $(4.6 \%)$, and outdoor sports facility (1.1\%). Demographic differences in the likelihood of engaging in physical activity in the top three locations (home/garage, in neighborhood, park/trail) were examined. 
Individuals who worked part-time were more than twice as likely $(\mathrm{OR}=2.12,95 \% \mathrm{CI}=$ $[1.02,4.41])$ and individuals who were not working were $83 \%$ less likely $(\mathrm{OR}=0.17,95 \% \mathrm{CI}=$ $[0.060 .51])$ to engage in physical activity at home or in their garage than individuals who worked full-time. Hispanics were about two-thirds less likely to engage in physical activity on sidewalks and roads in their neighborhoods than non-Hispanics $(\mathrm{OR}=0.34,95 \% \mathrm{CI}=[0.18,0.66])$. Also, individuals who worked part-time $(\mathrm{OR}=0.33,95 \% \mathrm{CI}=[0.12,0.91])$ or were in school only $(\mathrm{OR}$ $=0.34,95 \% \mathrm{CI}=[0.16,0.74])$ were about two-thirds less likely to engage in physical activity on sidewalks and roads in their neighborhoods than individuals who worked full-time. Furthermore, individuals who had annual household incomes of $\$ 27,000-\$ 59,999(\mathrm{OR}=4.25,95 \% \mathrm{CI}=[1.7$,$10.55])$ or $\$ 60,000-\$ 99,999(\mathrm{OR}=4.18,95 \% \mathrm{CI}=[1.61,10.84])$ or more $\$ 100,000(\mathrm{OR}=4.24$, $95 \% \mathrm{CI}=[1.87,10.45])$ than were more than four times as likely to engage in physical activity on sidewalks and roads in their neighborhoods than individuals whose annual household incomes were less than $\$ 27,000$. Demographic differences in the likelihood of engaging in physical activity on a park or trail also emerged. Adults who were ages $40-59$ were more than twice as likely to report physical activity at a park or trail than adults ages $18-39(\mathrm{OR}=2.28,95 \% \mathrm{CI}=$ $[1.08,4.80])$. Also, Hispanic adults were $62 \%$ less likely to report physical activity at a park or trail than non-Hispanic adults $(\mathrm{OR}=0.38,95 \% \mathrm{CI}=[0.15,0.97])$.

\section{Discussion}

This study is one of the first known empirical investigations to examine the early effects of the COVID-19 pandemic on physical activity levels among U.S. adults. The baseline survey period represented the period of time (early-COVID-19, April 3-May 5, 2020) when states were under the most restrictive of the "Shelter-in-Place" and "Stay-at-home" orders, which prohibited most non-essential travel outside of the home. Results indicated that physical activity at all levels 
(vigorous, moderate, and walking) substantially decreased during this period as compared to the month (pre-COVID-19, February 2020) immediately leading up to the declaration of the COVID-19 National Emergency in the U.S. However, declines in physical activity levels during the early-COVID-19 period were not universal for everyone. Adults who were not working, represented lower income households, or were Hispanic experienced sharper decreases in physical activity. These disparities indicate that certain population sub-groups may be hit harder by the COVID-19 pandemic in terms of its impact on health-related behaviors. Given the potential for increased risk of obesity, diabetes, and other chronic diseases due to prolonged physical inactivity; immediate communication, programmatic, and policy efforts should be directed towards promoting physical activity in these vulnerable groups during the next few months of the pandemic.

Overall, physical activity levels declined dramatically during the early-COVID-19 period. Observed changes ranged from a 33\% decrease for walking physical activity to an almost $47 \%$ decrease for moderate intensity physical activity. These values are similar in size to percent changes in the range of $32 \%-48 \%$ found for overall physical activity on recent self-report surveys of U.S. adults for the late March to early April period $(11,12)$. In contrast, data from wearable activity tracking devices collected during the same time frame have found smaller decreases in physical activity levels ranging from $7 \%-12 \%(27,28)$. However, it should be noted that data from wearable tracking devices are often reported in terms of numbers of steps and do not disentangle activity intensity levels (vigorous, moderate, light). In the current study, the smallest decreases were observed for minutes of walking per week, which may more closely approximate overall steps than higher intensity activities (such as resistance exercises or biking), which may not be captured by wearable devices. The larger reductions observed for moderate 
and vigorous intensity physical activity are most likely due to the closure of gyms/fitness facilities that provide cardiovascular and weight-lifting equipment, as well as vigorous intensity group exercise classes, such as HIIT (high-intensity interval training) classes. Although it is likely that some individuals may attempt to replace these resources with online/streaming fitness classes or available equipment at home, the overall trends suggest that vigorous intensity physical activities are bearing the largest impact of the COVID-19 gym/fitness facility closures.

Reductions in vigorous intensity physical activity differed across sub-groups characterized by variations in current work/school status during the early-COVID-19 period (April 3- May 5, 2020). Adults who were not working (and not in school) and adults who were in school only (and not working) reported larger reductions in vigorous intensity physical activity. In contrast, adults who were working full-time or part-time seemed to be more buffered from the impact of COVID-19 on their vigorous physical activity levels. The location data during the early-COVID-19 period suggest that individuals who were not working or in school were less likely to perform physical activity at home or in their garage, or on the sidewalks and roads in their neighborhood. One potential explanation for the effects on the sub-group reporting being in school is that those individuals may have been previously residing in university dorms or other on-campus living arrangements but are now living at home, and no longer have access to university-based sports or gyms/fitness facilities. The larger declines in vigorous intensity physical activity for adults who are not working may reflect them no longer being able to access workplace-based exercise facilities, not being able to afford the purchase of home exercise equipment or access to online streaming exercise classes, lack of motivation to continue vigorous activity due to being laid off, or childcare and supervision of homeschooling requirements that leave little time for participating in structured vigorous activities. Regardless, the substantial 
declines in vigorous intensity physical activity for individuals who are not working or who are in school highlights vulnerable groups who may experience more long-lasting physical health effects more affordable and easily accessible opportunities for home- or neighborhood-based structured higher-intensity exercise become available.

Data further suggest that decreases in minutes of walking per week were larger in individuals living in lower income households and for Hispanics. A possible explanation for this decline is COVID-19-based elimination of transport- and occupational-based walking among individuals who previously traveled to work all or part of the time by walking or public transportation, or who previously engaged in walking while at work due to the nature of their job position. Location data during the early-COVID-19 period indicate that Hispanics and individuals from lower income households were less likely to report engaging in physical activity on the sidewalks and roads in their neighborhood. Several reasons may account for this finding, such as the possibility that transport- or occupational-based physical activity is not being replaced by leisure walking in the neighborhood. Also, it is possible that streets in lower-income and Hispanic neighborhoods have poorer sidewalk availability and quality, greater traffic volume, or higher crime, which can deter leisure walking $(29,30)$. Location data also show that Hispanics were less likely to report engaging in physical activity in parks or on trails, which also could be due to lower availability of these amenities in Hispanic neighborhoods(31-33) or regional differences in park closures that disproportionally affect areas of the U.S. with greater concentration of Hispanics (such as California (34)). Patterns observed here suggest that disparate impacts of the COVID-19 pandemic on lower income and ethnic minority groups reach beyond viral exposures and mortality (35-37), and extend into health-related behaviors that may have long-lasting consequences for other chronic diseases. 
Strengths of the study included the timeliness of the baseline survey during the earlyCOVID-19 period, the use of standardized physical activity measures, and wide geographic and sociodemographic representation. However, there were some limitations. The use of a retrospective approach to assess pre-COVID-19 levels of physical activity could have introduced various reporting errors and biases. However, the within-subject design attempts to limit these concerns by only comparing each participant to him- or herself. Furthermore, about $31 \%$ of the enrolled participants had not yet completed the full baseline survey by the time of data analysis. Participants were allowed to postpone the completion of the baseline survey to a time that was more convenient for them. Lastly, the sample largely consisted of younger adults who were female and more highly educated. Findings may not extend to older adults, males, or those who have not attended college.

\section{Conclusions}

In summary, results from this study found that physical activity among U.S. adults decreased dramatically during the first full month of the "Shelter-in-Place" and "Stay-at-home" order issued by states during the COVID-19 pandemic. Although these restrictions were necessary in order to slow the spread of the virus and allow healthcare facilities to build capacity, there may be unintended consequences on other health-related behaviors such as physical activity. Disparate impacts of the COVID-19 pandemic on the physical activity levels of potentially vulnerable sub-groups, including individuals who are not working, live in lower income households, or are Hispanic, underscore the need for population-specific physical activity programs and policies over the next several months to years as the pandemic continues. 
Acknowledgements: This study was funded by National Institutes of Health grants

R01HL119255 and U01HL146327. We would like to thank Amy Nguyen for her assistance with participant recruitment and data collection. We would also like to thank Kayla Nuss for helping design the baseline physical activity survey.

Conflict of Interest: There are no conflicts of interest to declare. The results of the present study do not constitute endorsement by ACSM. The results of the study are presented clearly, honestly, and without fabrication, falsification, or inappropriate data manipulation. 


\section{References}

1. Centers for Disease Control and Prevention. Coronavirus Disease 2019 (COVID-19)

Cases in the U.S. Available from: https://www.cdc.gov/coronavirus/2019-ncov/casesupdates/cases-in-us.html.

2. FINRA. State "Shelter-in-Place" and "Stay-at-Home" Orders. Available from: https://www.finra.org/rules-guidance/key-topics/covid-19/shelter-in-place.

3. Education Commission of the States. COVID Response May 6.

4. UNESCO. COVID-19 Educational Disruption and Response.

5. U.S. Department of Health and Human Services. Physical Activity Guidelines for Americans. 2018.

6. Du Y, Liu B, Sun Y, Snetselaar LG, Wallace RB, Bao W. Trends in adherence to the Physical Activity Guidelines for Americans for aerobic activity and time spent on sedentary behavior among US adults, 2007 to 2016. JAMA Netw Open. 2019;2(7):e197597-e.

7. Zenko Z, Willis EA, White DA. Proportion of adults meeting the 2018 physical activity guidelines for Americans according to accelerometers. Front Public Health. 2019;7:135.

8. Pinto AJ, Dunstan DW, Owen N, Bonfá E, Gualano B. Combating physical inactivity during the COVID-19 pandemic. Nat Rev Rheumatol. 2020:1-2.

9. Lippi G, Henry BM, Sanchis-Gomar F. Physical inactivity and cardiovascular disease at the time of coronavirus disease 2019 (COVID-19). Eur J Prev Cardiol. 2020:2047487320916823.

10. Rodriguez M. Latest Data Shows U.S. Health Club Industry Serves 70.2 Million: International Health, Racquet and Sportsclub Association; 2018. Available from: 
https://www.ihrsa.org/about/media-center/press-releases/latest-data-shows-u-s-health-clubindustry-serves-70-2-million/.

11. Meyer J, McDowell, C., Lansing, J., Brower, C., Smith, L., Tully, M., \& Herring, M. Changes in physical activity and sedentary behaviour due to the COVID-19 outbreak and associations with mental health in 3,052 US adults. Cambridge Open Engage. doi: 10.33774/coe2020-h0b8g.

12. Evidation, Inc. COVID-19 Pulse: Delivering regular insights on the pandemic from a 150,000+ person connected cohort. Available from: https://evidation.com/news/covid-19-pulsefirst-data-evidation.

13. Halabchi F, Ahmadinejad Z, Selk-Ghaffari M. COVID-19 Epidemic: Exercise or Not to Exercise; That is the Question! Asian J Sports Med. 2020.

14. Yan Z, Spaulding HR. Extracellular superoxide dismutase, a molecular transducer of health benefits of exercise. Redox Biol. 2020:101508.

15. Bélisle M, Roskies E, Lévesque J-M. Improving adherence to physical activity. Health Psychol. 1987;6(2):159.

16. Redcap Project. Available from: http://project-redcap.org.

17. Harris PA, Taylor R, Thielke R, Payne J, Gonzalez N, Conde JG. Research electronic data capture (REDCap) — a metadata-driven methodology and workflow process for providing translational research informatics support. J Biomed Inform. 2009;42(2):377-81.

18. National Jewish Health. Coronavirus: Information \& Resources. Available from: https://www.nationaljewish.org/patients-visitors/patient-info/important-updates/coronavirusinformation-and-resources/news-and-research/covid-19-research. 
19. Bauman A, Ainsworth BE, Bull F, Craig CL, Hagströmer M, Sallis JF, et al. Progress and pitfalls in the use of the International Physical Activity Questionnaire (IPAQ) for adult physical activity surveillance. J Phys Act Health. 2009;6(s1):S5-S8.

20. Brown W, Trost S, Bauman A, Mummery K, Owen N. Test-retest reliability of four physical activity measures used in population surveys. J Sci Med Sport. 2004;7(2):205-15.

21. Ekelund U, Sepp H, Brage S, Becker W, Jakes R, Hennings M, et al. Criterion-related validity of the last 7-day, short form of the International Physical Activity Questionnaire in Swedish adults. Public Health Nutr. 2006;9(2):258-65.

22. Mäder U, Martin BW, Schutz Y, Marti B. Validity of four short physical activity questionnaires in middle-aged persons. Med Sci Sports Exerc. 2006;38(7):1255-66.

23. Guidelines for data processing and analysis of the International Physical Activity Questionnaire (IPAQ) - Short and Long Forms Available from: https://docs.google.com/viewer?a=v\&pid=sites\&srcid=ZGVmYXVsdGRvbWFpbnx0aGVpcGF xfGd4OjE0NDgxMDk3NDU1YWRIZTM.

24. Thompson EP, Roman RJ, Moskowitz GB, Chaiken S, Bargh JA. Accuracy motivation attenuates covert priming: The systematic reprocessing of social information. J Pers Soc Psychol. 1994;66(3):474.

25. Olejnik S, Algina J. Generalized eta and omega squared statistics: measures of effect size for some common research designs. Psychol Methods. 2003;8(4):434.

26. Steiger JH. Beyond the F test: effect size confidence intervals and tests of close fit in the analysis of variance and contrast analysis. Psychol Methods. 2004;9(2):164.

27. Fitbit, Inc. The Impact Of Coronavirus On Global Activity [5/13/20]. Available from: https://blog.fitbit.com/covid-19-global-activity/. 
28. Withings, Inc. Data: Quarantine Isn’t Making Us Gain Weight or Slow Down. Available from: https://blog.withings.com/2020/05/01/data-quarantine-isnt-making-us-gain-weight-orslow-down/.

29. Silfee VJ, Rosal MC, Sreedhara M, Lora V, Lemon SC. Neighborhood environment correlates of physical activity and sedentary behavior among Latino adults in Massachusetts. BMC Public Health. 2016;16(1):966.

30. Larsen BA, Pekmezi D, Marquez B, Benitez TJ, Marcus BH. Physical activity in Latinas: social and environmental influences. Womens Health. 2013;9(2):201-10.

31. Cronan MK, Shinew KJ, Stodolska M. Trail Use Among Latinos: Recognizing Diverse Uses Among A Specific Population. J Park Recreat Admi. 2008;26(1).

32. Derose KP, Han B, Williamson S, Cohen DA. Racial-ethnic variation in park use and physical activity in the City of Los Angeles. J Urban Health. 2015;92(6):1011-23.

33. Moore LV, Roux AVD, Evenson KR, McGinn AP, Brines SJ. Availability of recreational resources in minority and low socioeconomic status areas. Am J Prev Med. 2008;34(1):16-22. 34. California Department of Parks and Recreation. COVID-19 Closures 2020. Available from: https://www.parks.ca.gov/?page id=30355.

35. Centers for Disease Control and Prevention. Hospitalization Rates and Characteristics of Patients Hospitalized with Laboratory-Confirmed Coronavirus Disease 2019 - COVID-NET, 14 States, March 1-30, 2020 2020. Available from: https://www.cdc.gov/mmwr/volumes/69/wr/mm6915e3.htm?s_cid=mm6915e3_w. 36. California Department of Public Health. COVID-19 Race and Ethnicity Data 2020. Available from: https://www.cdph.ca.gov/Programs/CID/DCDC/Pages/COVID-19/RaceEthnicity.aspx. 
37. Centers for Disease Control and Prevention. COVID-19 in Racial and Ethnic Minority Groups 2019. Available from: https://www.cdc.gov/coronavirus/2019-ncov/need-extraprecautions/racial-ethnic-minorities.html. 


\section{Figure Captions}

Figure 1.

Panel A: Effects of time (pre-COVID-19 [Feb. 2020] vs. early-COVID-19 [April 3-May 5, 2020]) on minutes of vigorous intensity physical activity per week by work/school status (work full-time $n=129$, work part-time $n=59$, not working $n=23$, in school $n=38$ ). Estimated marginal means are presented for raw (untransformed) data adjusting for ethnicity (Hispanic vs. non-Hispanic). $\mathrm{n}=249$

Panel B: Effects of time (pre-COVID-19 [Feb. 2020] vs. early-COVID-19 [April 3-May 5, 2020]) on minutes of walking per week by annual household income category $(\$ 12,500-\$ 26,999$ $\mathrm{n}=35, \$ 27,000-\$ 59,999 \mathrm{n}=56, \$ 60,000-\$ 99,999 \mathrm{n}=41$, over $\$ 100,000 \mathrm{n}=91)$. Estimated marginal means are presented for raw (untransformed) data adjusting for ethnicity (Hispanic vs. non-Hispanic). $\mathrm{n}=223$

Panel C: Effects of time (pre-COVID-19 [Feb. 2020] vs. early-COVID-19 [April 3-May 5, 2020]) on minutes of walking per week by ethnicity (Hispanic $n=50$, non-Hispanic $n=196$ ). Estimated marginal means are presented for raw (untransformed) data adjusting for work/school status (during April 3-May 5, 2020). $\mathrm{n}=246$ 
Tables

Table 1: Descriptive Statistics for Sample on Demographic Characteristics

\begin{tabular}{|c|c|}
\hline Variable & $n(\%)$ \\
\hline \multicolumn{2}{|l|}{ Age Group } \\
\hline 18-39 years & $206(78.6)$ \\
\hline $40-59$ years & $40(15.3)$ \\
\hline$\geq 60$ years & $16(6.1)$ \\
\hline \multicolumn{2}{|l|}{ Sex } \\
\hline Male & $48(18.3)$ \\
\hline Female & $214(81.7)$ \\
\hline \multicolumn{2}{|l|}{ Ethnicity ${ }^{\mathbf{a}}$} \\
\hline Hispanic & $53(20.4)$ \\
\hline Non-Hispanic & $207(79.6)$ \\
\hline \multicolumn{2}{|l|}{ Race $^{b}$} \\
\hline White & $164(64.3)$ \\
\hline Black & $9(3.5)$ \\
\hline Asian & $45(17.6)$ \\
\hline Mixed race & $19(7.5)$ \\
\hline Other & $18(7.1)$ \\
\hline \multicolumn{2}{|l|}{ Education } \\
\hline Completed high school/GED & $7(2.7)$ \\
\hline Some college & $17(6.5)$ \\
\hline College graduate & $104(39.7)$ \\
\hline Graduate school degree & $134(51.1)$ \\
\hline \multicolumn{2}{|l|}{ Current Work/School Status ${ }^{c}$} \\
\hline Working full-time & $133(51.0)$ \\
\hline Working part-time & $62(23.8)$ \\
\hline Not working & $26(10.0)$ \\
\hline In School & $40(15.3)$ \\
\hline \multicolumn{2}{|l|}{ Annual Household Incomed } \\
\hline Less than $\$ 27,000$ & $39(16.5)$ \\
\hline$\$ 27,000-\$ 59,999$ & $57(24.2)$ \\
\hline$\$ 60,000-\$ 99,999$ & $45(19.1)$ \\
\hline$\$ 100,000$ or more & $95(40.3)$ \\
\hline \multicolumn{2}{|l|}{ Weight Categories ${ }^{\mathrm{e}}$} \\
\hline Underweight & $7(2.8)$ \\
\hline Normal weight & $142(55.9)$ \\
\hline Overweight & $72(28.3)$ \\
\hline Obese & $33(13.0)$ \\
\hline
\end{tabular}


Table 2: Descriptive Statistics and Within-Subjects Repeated Measures ANOVAs for Physical Activity Variables

\begin{tabular}{|c|c|c|c|c|c|c|c|c|}
\hline & \multicolumn{2}{|c|}{$\begin{array}{c}\text { Pre-COVID-19 } \\
\text { (Feb. 2020) }\end{array}$} & \multicolumn{2}{|c|}{$\begin{array}{c}\text { Early-COVID-19 } \\
\text { (April 3-May 5, 2020) }\end{array}$} & \multirow[b]{2}{*}{$d f$} & \multirow[b]{2}{*}{$\boldsymbol{F}$} & \multirow[b]{2}{*}{$p$} & \multirow[b]{2}{*}{$\eta_{p}^{2}$} \\
\hline & $n$ & $M(S D$ & $n$ & $M(S D)$ & & & & \\
\hline Vigorous min/week & 255 & $166.9(164.9)$ & 256 & $105.2(137.2)$ & 1,247 & 24.9 & $<.001$ & .091 \\
\hline Moderate min/week & 256 & $181.7(248.6)$ & 259 & $97.6(136.4)$ & 1,250 & 48.8 & $<.001$ & .163 \\
\hline Walking min/week & 256 & $315.5(320.4)$ & 252 & $211.1(251.3)$ & 1,253 & 29.7 & $<.001$ & .105 \\
\hline
\end{tabular}

$\eta_{\mathrm{p}}{ }^{2}=$ effect size (partial eta squared). Raw (untransformed) means are presented for ease of interpretation. Repeated Measures ANOVAs are reported for squared root-transformed data. 
Table 3: Mixed Model Repeated-Measures Analysis of Variance (ANOVA) Examining the Effects of Time (pre-COVID-19 vs. earlyCOVID-19) on Minutes of Vigorous Intensity Physical Activity per Week by Ethnicity, School/Work Status, and Annual Household Income

\begin{tabular}{|c|c|c|c|c|c|c|c|c|}
\hline & \multicolumn{4}{|c|}{ Model 1} & \multicolumn{4}{|c|}{ Model 2} \\
\hline & $d f$ & $\boldsymbol{F}$ & $p$ & $\eta_{\mathrm{p}}^{2}$ & $d f$ & $\boldsymbol{F}$ & $p$ & $\eta_{\mathrm{p}}^{2}$ \\
\hline Time (WS) & 1,221 & 32.09 & $<.001$ & .127 & 1,244 & 45.8 & $<.001$ & .158 \\
\hline Hispanic (BS) & 1,221 & 2.03 & .156 & .009 & 1,244 & 2.08 & .151 & .008 \\
\hline Annual Household Income (BS) & 3,221 & 2.00 & .115 & .026 & & & & \\
\hline Work/School Status (BS) & & & & & 3,244 & 2.41 & .067 & .029 \\
\hline Time $x$ Hispanic & 1,221 & 0.77 & .380 & .003 & 1,244 & 0.76 & .384 & .003 \\
\hline Time x Annual Household Income & 3,221 & 0.86 & .465 & .011 & & & & \\
\hline Time $x$ Work/School Status & & & & & 3,244 & 3.46 & .017 & .041 \\
\hline
\end{tabular}

$\mathrm{n}=226$ for Model 1 and $\mathrm{n}=249$ for Model $2 . \eta_{\mathrm{p}}{ }^{2}=$ effect size (partial eta squared). WS = within-subject effect. BS $=$ between-subject effect. Vigorous intensity physical activity (min/week) was square root transformed. pre-COVID-19 (Feb 2020) and early-COVID-19 (April 3 - May 5, 2020). Hispanic (vs. non-Hispanic). Annual household income (\$12,500-\$26,999, \$27,000-\$59,999, \$60,000$\$ 99,999$, more than $\$ 100,000)$. Work/school status (work full-time, work part-time, not working, in school). 
Table 4: Mixed Model Repeated-Measures Analysis of Variance (ANOVA) Examining the Effects of Time (pre-COVID-19 vs. earlyCOVID-19) on Minutes of Moderate Intensity Physical Activity per Week by Ethnicity, School/Work Status, and Annual Household Income

\begin{tabular}{|c|c|c|c|c|c|c|c|c|}
\hline & \multicolumn{4}{|c|}{ Model 1} & \multicolumn{4}{|c|}{ Model 2} \\
\hline & $d f$ & $\boldsymbol{F}$ & $p$ & $\eta_{\mathrm{p}}^{2}$ & $d f$ & $\boldsymbol{F}$ & $p$ & $\eta_{\mathrm{p}}^{2}$ \\
\hline Time (WS) & 1,224 & 32.27 & $<.001$ & .126 & 1,248 & 19.63 & $<.001$ & .073 \\
\hline Hispanic (BS) & 1,225 & 0.01 & .946 & $<.001$ & 1,248 & 0.11 & .736 & $<.001$ \\
\hline Annual Household Income (BS) & 3,224 & 0.32 & .813 & .004 & & & & \\
\hline Work/School Status (BS) & & & & & 3,248 & 2.97 & .033 & .035 \\
\hline Time $x$ Hispanic & 1,224 & 0.41 & .524 & .002 & 1,248 & 0.22 & .638 & .001 \\
\hline Time x Annual Household Income & 3,224 & 2.54 & .058 & .033 & & & & \\
\hline Time x Work/School Status & & & & & 3,248 & 0.49 & .687 & .006 \\
\hline
\end{tabular}

$\mathrm{n}=229$ for Model 1 and $\mathrm{n}=253$ for Model $2 . \eta_{\mathrm{p}}{ }^{2}=$ effect size (partial eta squared). WS = within-subject effect. BS $=$ between-subject effect. Moderate intensity physical activity (min/week) was square root transformed. pre-COVID-19 (Feb 2020) and early-COVID-19 (April 3 - May 5, 2020). Hispanic (vs. non-Hispanic). Annual household income $(\$ 12,500-\$ 26,999, \$ 27,000-\$ 59,999$, $\$ 60,000-$ $\$ 99,999$, more than $\$ 100,000)$. Work/school status (work full-time, work part-time, not working, in school). 
Table 5: Mixed Model Repeated-Measures Analysis of Variance (ANOVA) Examining the Effects of Time (pre-COVID-19 vs. earlyCOVID-19) on Minutes of Walking per Week by Ethnicity, School/Work Status, and Annual Household Income

\begin{tabular}{|c|c|c|c|c|c|c|c|c|}
\hline & \multicolumn{4}{|c|}{ Model 1} & \multicolumn{4}{|c|}{ Model 2} \\
\hline & $d f$ & $\boldsymbol{F}$ & $p$ & $\eta_{\mathrm{p}}^{2}$ & $d f$ & $\boldsymbol{F}$ & $p$ & $\eta_{\mathrm{p}}^{2}$ \\
\hline Time (WS) & 1,218 & 27.57 & $<.001$ & .112 & 1,241 & 24.74 & $<.001$ & .093 \\
\hline Hispanic (BS) & 1,218 & 5.41 & .021 & .024 & 1,241 & 5.34 & .022 & .022 \\
\hline Annual Household Income (BS) & 3,218 & 0.35 & .792 & .005 & & & & \\
\hline Work/School Status (BS) & & & & & 3,241 & .882 & .451 & .011 \\
\hline Time $x$ Hispanic & 1,218 & 3.69 & .056 & .017 & 1,241 & 5.02 & .026 & .020 \\
\hline Time x Annual Household Income & 3,218 & 2.77 & .042 & .037 & & & & \\
\hline Time x Work/School Status & & & & & 3,241 & 1.92 & .127 & .023 \\
\hline
\end{tabular}

$\mathrm{n}=223$ for Model 1 and $\mathrm{n}=246$ for Model $2 . \eta_{\mathrm{p}}{ }^{2}=$ effect size (partial eta squared). WS = within-subject effect. BS $=$ between-subject effect. Walking (min/week) was square root transformed. pre-COVID-19 (Feb 2020) and early-COVID-19 (April 3 - May 5, 2020).

Hispanic (vs. non-Hispanic). Annual household income (\$12,500-\$26,999, \$27,000-\$59,999, \$60,000-\$99,999, more than \$100,000).

Work/school status (work full-time, work part-time, not working, in school). 
Figures

Figure 1.

Panel A:

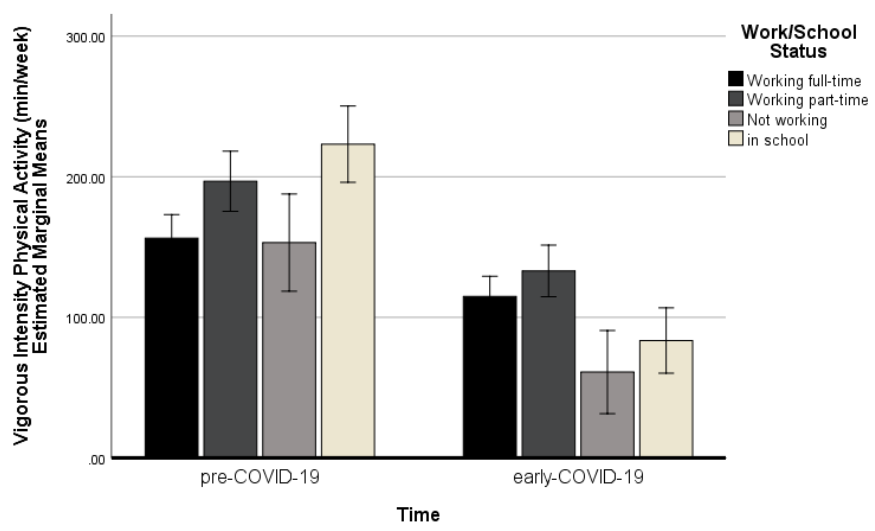

Panel B:

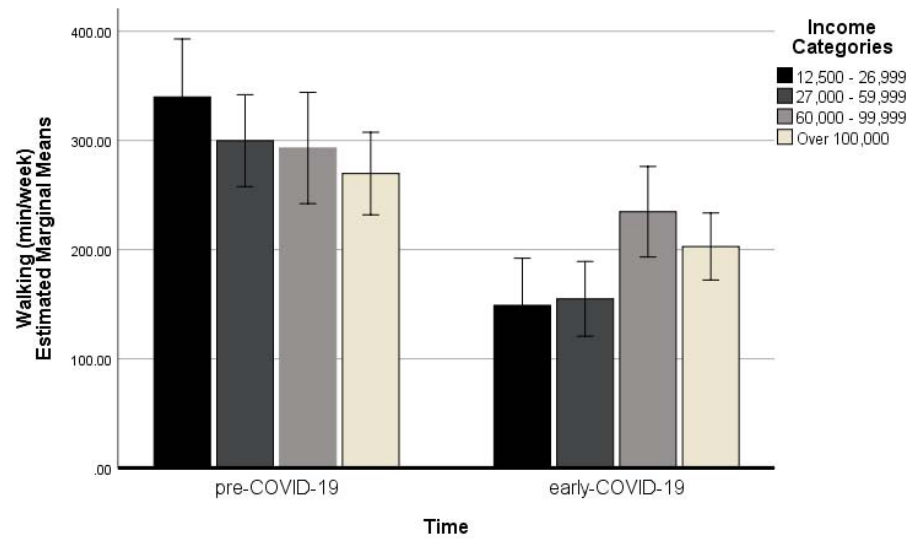

Panel C:

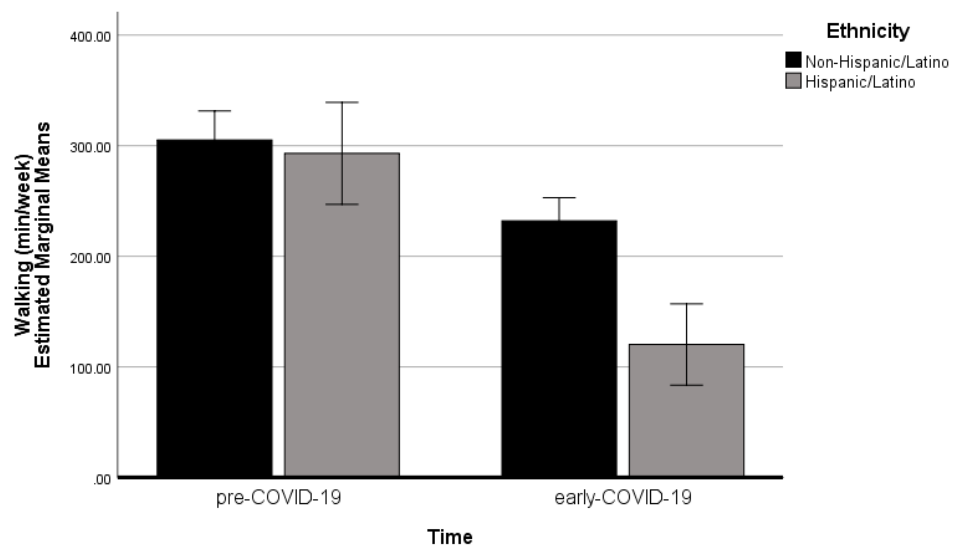

